\title{
Reduction of heat sink common-mode currents in switching mode power supply circuits
}

\author{
J. Kulanayagam, J. H. Hagmann, K. F. Hoffmann, and S. Dickmann \\ Helmut-Schmidt-University/University of the Federal Armed Forces Hamburg, Germany
}

\begin{abstract}
In this paper, a new filter design for a heat sink is presented. The parasitic couplings between electric power devices and the heat sink are responsible for common-mode currents. The main focus is on the reduction of these currents to reduce the heat sink radiation. For this purpose a new filter design is proposed. In addition, experimental results are shown to validate the proposed filter.
\end{abstract}

\section{Introduction}

In general, power-switching semiconductors (MOSFET's/IGBT's) are mounted on heat sinks to keep the power semiconductors within a given temperature range. A parasitic capacitance can be formed between the case of the semiconductors and the heat sink. The insulating thermal compound (Damiano et al., 2004) is the dielectric of this capacitance. It is shown as isolator 1 in Fig. 1.

Common-mode EMI is usually the result of parasitic effects (Tihanyi, 1995). Due to these capacitances commonmode currents are generated in Switching Mode Power Supply (SMPS) circuits. In consequence, these currents could violate the EMC standards by their radiation. Therefore, the heat sink should be well designed.

The drain-source voltage variation increases by decreasing the commutation time of the transistor and by increasing the insulation voltage level. Consequently, a high number of harmonic components are generated which makes the heat sink an EMI generator (Sinclair et al., 1993).

The shape and the dimension of the heat sinks play a major role in the power electronic system design. In general, as the size of the heat sink increases, the radiation efficiency also increases. However, the maximum radiation will take place

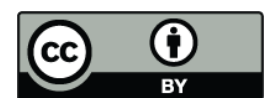

Correspondence to: J. Kulanayagam (kulanayagam@hsu-hamburg.de) at different frequencies depending on the heat sink geometry (Brench, 1994), (Das and Roy, 1998).

In particular, the heat sink can become an efficient antenna when resonant phenomena occur (Damiano et al., 2004). This paper investigates the origin of common-mode currents generated by SMPS and shows a filter design to reduce common-mode currents of ungrounded heat sinks and an experimental setup for validating the input impedance and the heat sink radiation.

\section{EMI generator and heat sink radiation}

A parasitic capacitance $C_{\mathrm{dh}}$ (see Fig. 2) exists between the metal case of the semiconductors and the heat sink. The insulating thermal compound acts as a dielectric for this capacitance. Due to this capacitance and the time varying drainsource voltage a noise current $i_{\text {noise }}$ can be calculated according to the following equation, as a first approach for low frequencies and $C_{\mathrm{dh}} \gg C_{\mathrm{hp}} \gg C_{\mathrm{hg}}$

$i_{\text {noise }}(t)=C_{\mathrm{dh}} \cdot \frac{d u_{\mathrm{ds}}}{d t}$,

where $d u_{\mathrm{ds}} / \mathrm{dt}$ is the time derivative of the drain-source voltage. $C_{\mathrm{dh}}$ can reach approximately $100 \mathrm{pF}$ (Tihanyi, 1995). This noise current $i_{\text {noise }}$ is split into the conducted EMI current $i_{\mathrm{c}}$ and the radiated EMI current $i_{\mathrm{h}}$

$i_{\text {noise }}(t)=i_{\mathrm{h}}(t)+i_{\mathrm{c}}(t)$.

The noise current paths through the heat sink in AC/DC converter are shown in Fig. 2 as an example.

In order to reduce the radiated emissions of the heat sink, it may be grounded. Consequently, the conducted EMI current $i_{\mathrm{c}}$ raises in the power supply (Tihanyi, 1995). The results of the previous works (Li et al., 1993; Radhakrishnan et al., 2000; Archambeault et al., 2001), have proved that grounding a heat sink can reduce radiated emissions. 


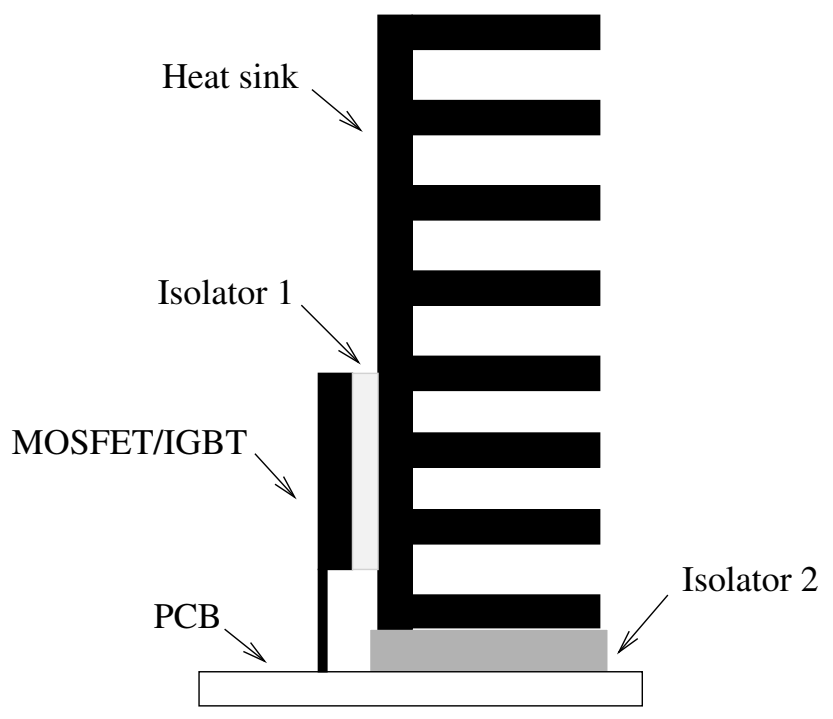

Fig. 1. Schematic representaion of parasitic capacitance.

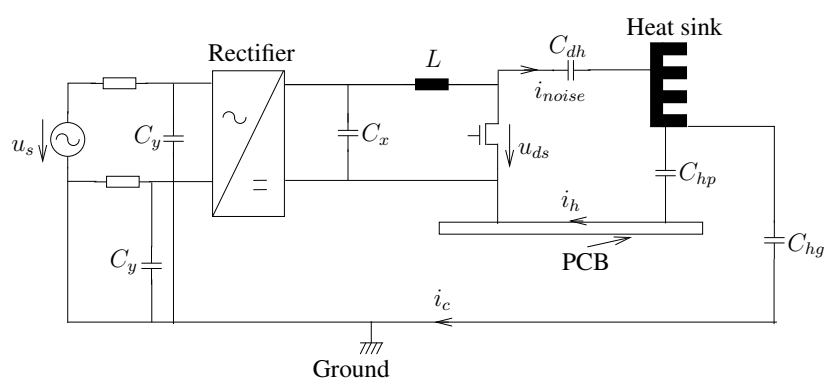

Fig. 2. Noise current paths through the heat sink in an AC/DC converter.

The noise current loops are shown in Fig. 2. The current path $i_{\mathrm{c}}$ via $C_{\mathrm{hg}}$ and ground represents the common-mode current and the current path $i_{\mathrm{h}}$ via $C_{\mathrm{hp}}$ and the PCB is the heat sink current. If the heat sink is not grounded, the conducted EMI current $i_{\mathrm{c}}$ can be reduced but the radiated EMI current $i_{\mathrm{h}}$ to the SMPS raises (Felic and Evans, 2001).

An efficient method to reduce radiated emissions of a heat sink was introduced in a previous study (Nagel, 1999). By inserting a copper screen between the heat sink and the power device the capacitance can be minimized. This screen is applied as a shield for the heat sink. Furthermore, the sides of the screen are insulated with a well thermally conducting dielectric material. This screening design is illustrated in Fig. 4.

In this work the radiated EMI current via the heat sink (see Fig. 3) is considered.

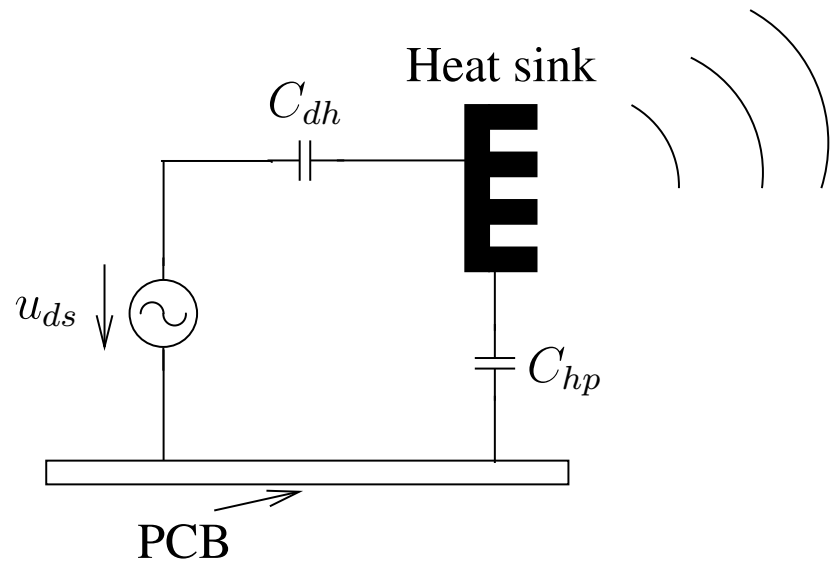

Fig. 3. Radiated EMI current via the heat sink.

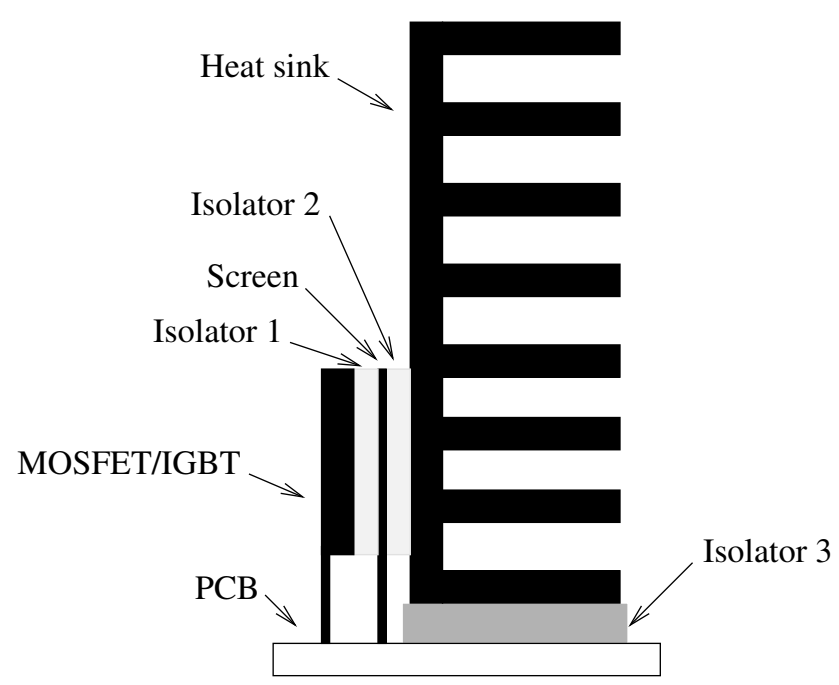

Fig. 4. Reduction of the common-mode current by screening.

\section{Filter design}

Due to the extensive use of digital information and communication equipment, EMC problems have increased. To mitigate this problem, ferrite cores are usually attached on the cable/ wire (see Fig. 5) to suppress electromagnetic noise emissions from digital information equipment (Samir and Fujiwara, 1999). This idea was integrated in the heat sink filter design to attenuate the heat sink radiated emissions.

The heat sink is extended with a metallic ring bar which has the same material characteristics like the heat sink. Furthermore, on the extended part of the heat sink a ferrite core is attached. The ferrite core is fixed between the heat sink and the power device. This is illustrated in Fig. 6.

The high frequency current is attenuated due to the ferrite core. This current can be calculated by the following equation : 


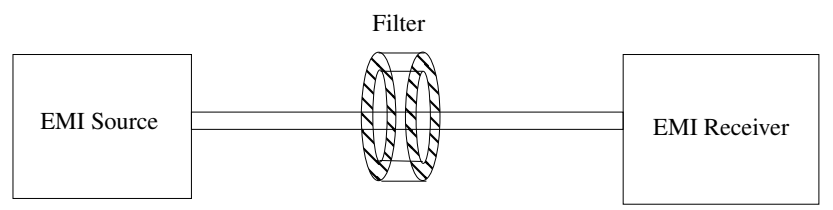

Fig. 5. Block diagram of the filter design.

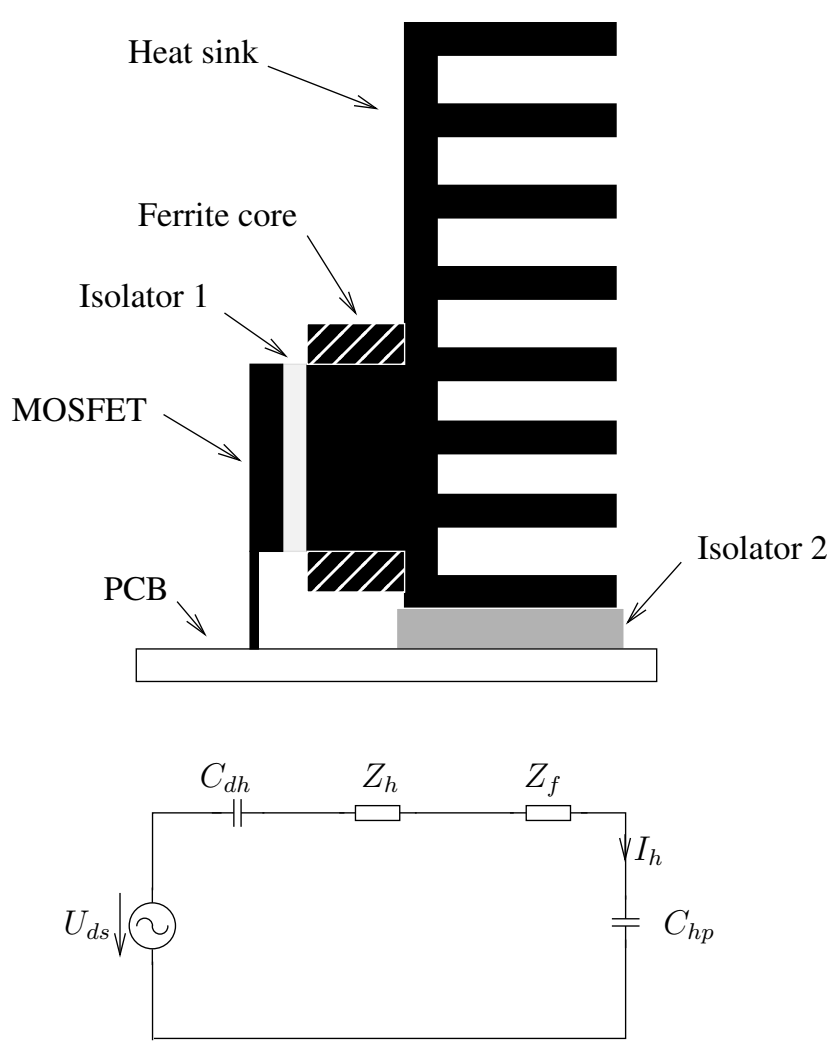

Fig. 6. Schematic representation of filter design and equivalent circuit.

$I_{\mathrm{h}}=\frac{U_{\mathrm{ds}}}{Z}$.

$I_{\mathrm{h}}$ is the heat sink current and $U_{\mathrm{ds}}$ is the drain-source voltage of the transistor and $Z$ is the impedance of the network.

The initial heat sink setup of Fig. 1 changes to the filter design of Fig. 6. An additional impedance $Z_{\mathrm{f}}$ has to be added in series to the resonant circuit. $Z_{\mathrm{f}}$ is the impedance of the ferrite core.

This new design has a higher heat transfer resistance than the original setup, but it has a lesser heat transfer resistance than the screening design.

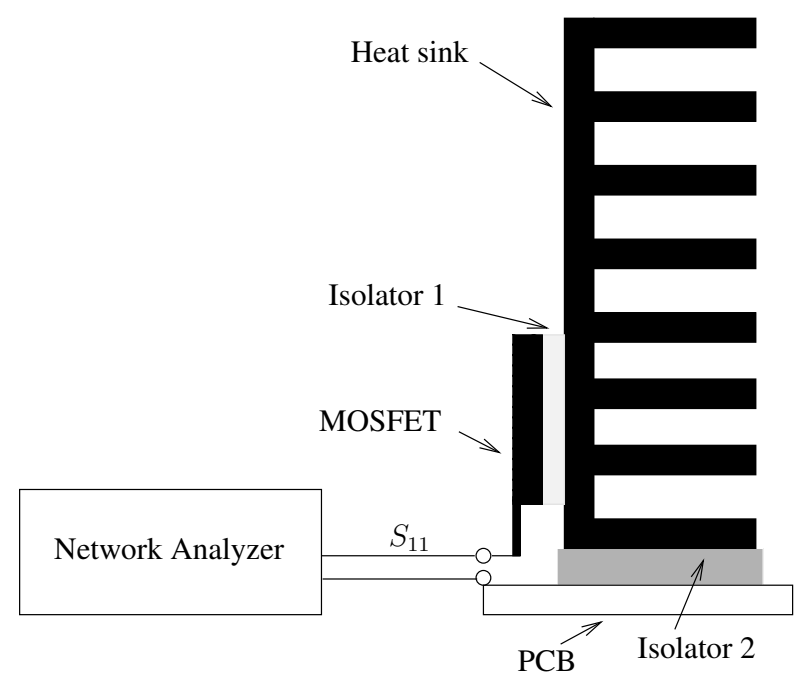

Fig. 7. Measurement setup for the input impedance of the reference heat sink design.

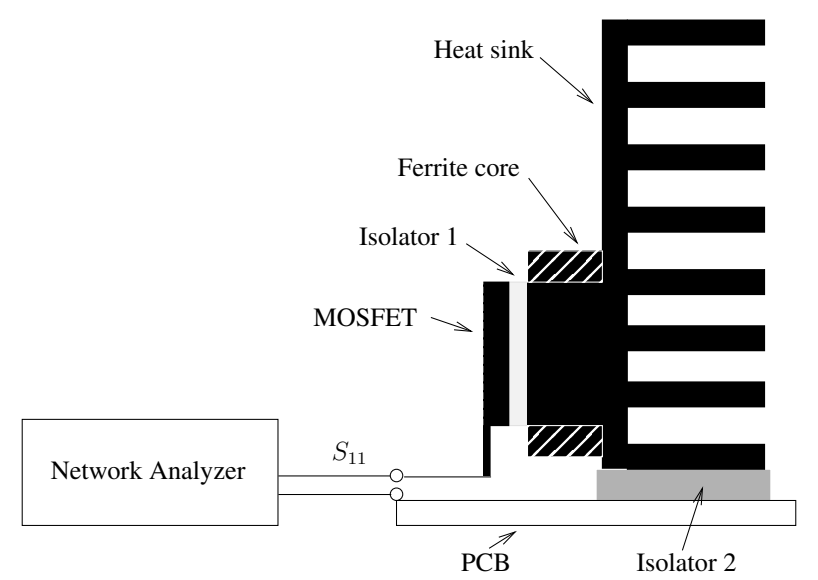

Fig. 8. Measurement setup for the input impedance of the ferrite heat sink design.

\section{Input impedance of the heat sink}

\subsection{Experimental setup}

Three different configurations were necessary to analyze the input impedance of the heat sink designs. The first setup is an original heat sink design for measuring the input impedance as a reference. It was placed to the vector network analyzer to measure the scattering parameters $S_{11}$ (see Fig. 7). A second and third measurement setup were made for the extended heat sink design without ferrite core and with ferrite core, respectively. The scattering parameters $S_{11}$ of these setups were measured by a vector network analyzer (see Fig. 8).

From these scattering parameters of all three configurations, the input impedances were calculated by using (Diepenbrock et al., 2001) 


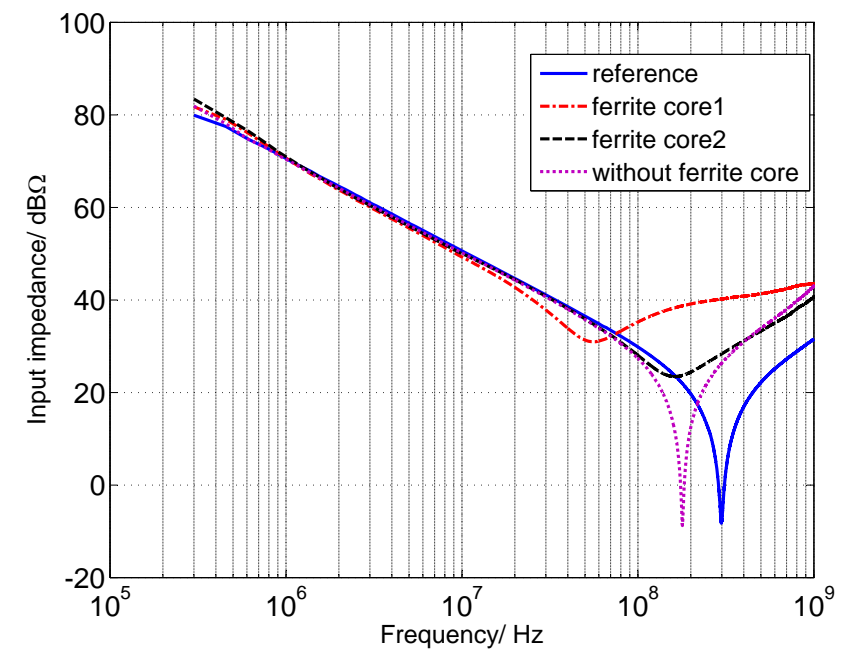

Fig. 9. Impedance of the reference, the enhanced heat sink with and without ferrite core.

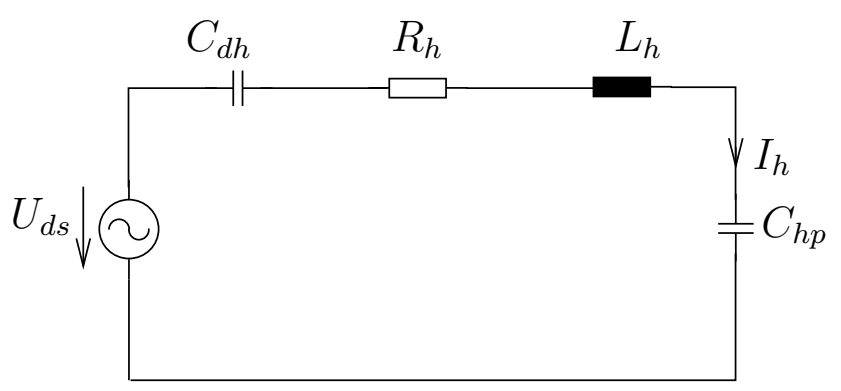

Fig. 10. Equivalent circuit for original heat sink design.

$Z_{\text {input }}=Z_{0} \cdot \frac{1+S_{11}}{1-S_{11}}$

where $Z_{\text {input }}$ is the input impedance to the network, the used normalization impedance $Z_{0}$ is $50 \mathrm{Ohms}$, and the measured scattering parameter data is $S_{11}$. In this work, the frequency range from $300 \mathrm{kHz}$ up to $1 \mathrm{GHz}$ was considered.

\subsection{Results and discussions}

The results of all three configurations are illustrated in Fig. 9. The curves of the first (reference heat sink design) and second (extended heat sink without ferrite core) configuration show, that the heat sink structures behave like series resonant circuits.

The result of the first configuration can be explained by the following equivalent circuit (see Fig. 10.)

The input impedance of the reference heat sink design can be expressed as:

$Z_{\text {input }}=\frac{1}{\mathrm{j} \omega C_{\mathrm{dh}}}+Z_{\mathrm{h}}+\frac{1}{\mathrm{j} \omega C_{\mathrm{hp}}}$.

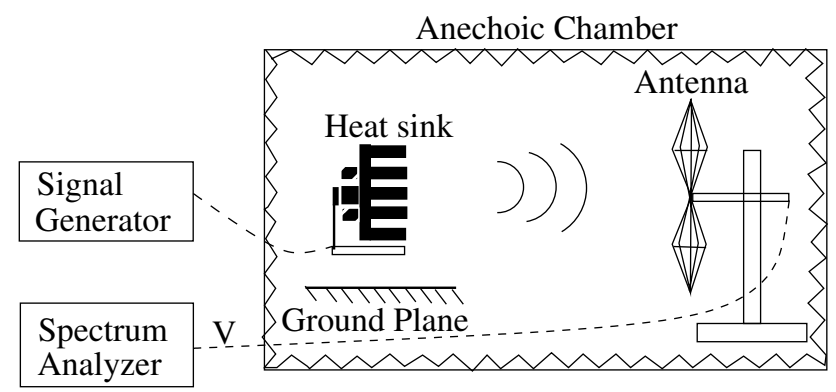

Fig. 11. Experimental setup for emission measurements.

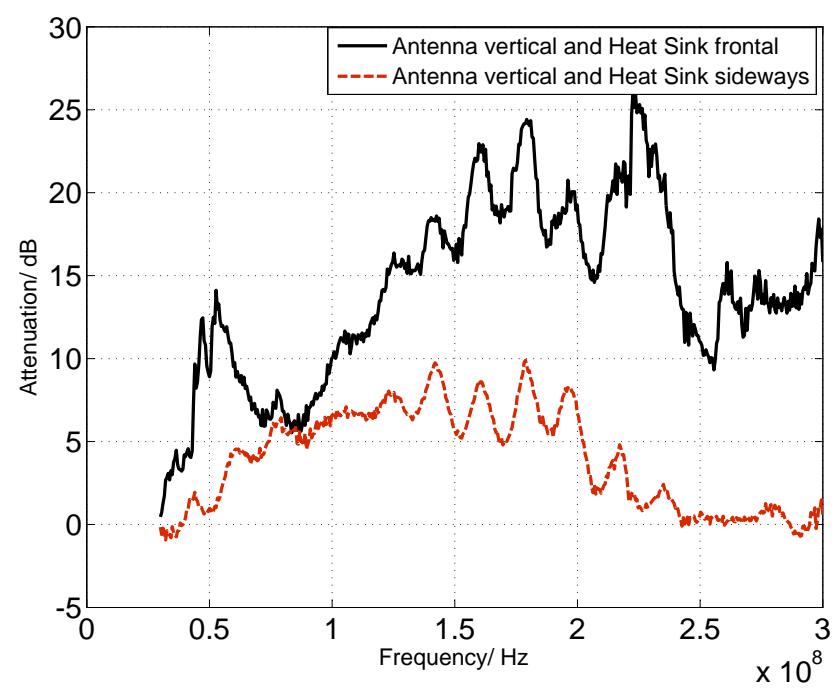

Fig. 12. Attenuation of the heat sink ferrite design in the frequency range for frontal and lateral measurements.

The impedance of the heat sink is given by

$Z_{\mathrm{h}}=R_{\mathrm{h}}+\mathrm{j} \omega L_{\mathrm{h}}$

The resonant frequency of the original heat sink design can be expressed as:

$f_{\text {res } 1}=\frac{1}{2 \pi \sqrt{L_{\text {loop }} \cdot C_{\text {loop }}}}$.

Up to the resonant frequency $f_{\text {res } 1}$, the input impedance behaves like a capacitance and above the resonant frequency $f_{\text {res1 }}$ the impedance behaves like an inductance. The resonant frequency $f_{\text {res2 } 2}$ of the second configuration was shifted into the lower $\mathrm{MHz}$ frequency range because the extended heat sink design has a larger current loop than the reference design. In other words, the second configuration has a larger inductance than the original heat sink setup. In consequence, the resonant frequency $f_{\text {res } 2}$ is smaller than the resonant frequency $f_{\text {res } 1}$. The resonant frequencies of the first and second configuration are $300 \mathrm{MHz}$ and $180 \mathrm{MHz}$, respectively. The capacitances of the third configuration (extended heat sink 
with ferrite core) are the same as first and second configuration. But the inductances of the heat sink are increased by the ferrite cores. This is because all two ferrite core consist of different materials and therefore different magnetic characteristics. The quality factor of the system is reduced by the ferrite core. Furthermore, it depends on the ferrite materials which is used.

The input impedance of the heat sink is increased because a ferrite core has a very large impedance due to its permeability. This means that the heat sink currents will be minimized by adding the ferrite core between the heat sink and power device and the heat sink radiation is attenuated by this filter.

\section{Radiation of the heat sink}

\subsection{Experimental setup}

In order to validate the attenuation of the heat sink radiated emissions of the proposed heat sink design an experimental test was done inside an anechoic chamber to avoid the presence of environmental EMI noises in EMI measurements. A simplified schematic of the experimental setup is shown in Fig. 11.

The RF signal generator was connected via a coaxial cable to the heat sink ferrite design. On the other side a biconical/ logarithmic periodic antenna receives the emitted electromagnetic fields from the heat sink. This received RF signal $V$ is detected by a spectrum analyzer.

From these RF signals of with and without ferrite core on the ungrounded heat sink, the attenuation of the heat sink radiated emissions is calculated using

$$
A_{\mathrm{hs}}(d B)=V_{\mathrm{w} / \mathrm{o} \text { Ferrite }}(d B \mu V)-V_{\mathrm{w} / \text { Ferrite }}(d B \mu V),
$$

where $A_{\mathrm{hs}}$ is the attenuation of the heat sink radiated emis-

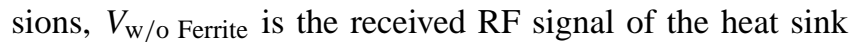
without ferrite core and $V_{\mathrm{w}}$ / Ferrite is the received RF signal of the heat sink with ferrite core. The measurement setups were configured forfrontal and lateral radiation. In this work, the frequency range from 20 up to $300 \mathrm{MHz}$ was considered.

\subsection{Results and discussions}

The attenuation of the heat sink radiation is calculated for frontal and lateral measurement of the heat sink is illustrated in Fig. 12. The maximum attenuation value and the average attenuation value of the frontal measurement of the heat sink are $25 \mathrm{~dB}$ and $15 \mathrm{~dB}$, respectively. Furthermore, the maximum attenuation value and the average attenuation value of the lateral measurement of the heat sink are $10 \mathrm{~dB}$ and $5 \mathrm{~dB}$, respectively.

\section{Conclusions}

In this paper a new filter design was introduced. It can be used to reduce the heat sink common-mode currents in Switching Mode Power Supply circuits. The validation of the input impedances of the heat sinks have verified that the input impedances can be increased at some frequencies up to $45 \mathrm{~dB}$ due to the ferrite core between the heat sink and the power device. Therefore, the heat sink current can be minimized. In addition, the radiated emissions from the heat sink are attenuated by the filter. Again, our proposed filter design reduces the heat sink noise currents and attenuates the heat sink radiated emissions.

\section{References}

Archambeault, B., Pratapneni, S., Zhang, L., and Wittwer, D.: Comparison of various numerical modeling tools against a standard problem concerning heat sink emissions, IEEE International Symposium on Electromagnetic Compatibility, 2, 1341-1346, 2001.

Brench, C.: Heatsink radiation as a function of geometry, IEEE International Symposium on Electromagnetic Compatibility, 105109, 1994.

Damiano, A., Gatto, G., Marongiu, I., and Piroddi, A.: A heat sink model for EMI resonance frequency determination, Power Electronics Specialists Conference, 1, 273-277, 2004.

Das, S. and Roy, T.: An investigation on radiated emissions from heatsinks, IEEE International Symposium on Electromagnetic Compatibility, 2, 784-789, 1998.

Diepenbrock, J., Archambeault, B., and Hobgood, L.: Improved grounding method for heat sinks of high speed processors, Electronic Components and Technology Conference, 993-996, 2001.

Felic, G. and Evans, R.: Study of heat sink EMI effects in SMPS circuits, IEEE International Symposium on Electromagnetic Compatibility, 1, 254-259, 2001.

Li, K., Lee, C., Poh, S., Shin, R., and Kong, J.: Application of FDTD method to analysis of electromagnetic radiation from VLSI heatsink configurations, IEEE Transactions on Electromagnetic Compatibility, 35, 204-214, 1993.

Nagel, A.: Leitungsgebundene Störungen in der Leistungselektronik: Entstehung, Ausbreitung und Filterung [Dissertation], Wissenschaftsverlag, Mainz, 1999.

Radhakrishnan, K., Wittwer, D., and Li, Y.-L.: Study of heatsink grounding schemes for $\mathrm{GHz}$ microprocessors, IEEE Conference on Electrical Performance of Electronic Packaging, 189-192, 2000.

Samir, A. and Fujiwara, O.: Calculation of load effect produced by ferrite core attached to wire above a ground plane, Asia Pacific Microwave Conference, 1, 1999.

Sinclair, A., Ferreira, J., and van Wyk, J.: A systematic study of EMI reduction by physical converter layout and suppressive circuits, International Conference on Industrial Electronics, Control, and Instrumentation, 2, 1059-1064, 1993.

Tihanyi, L.: Electromagnetic Compatibility in Power Electronics, J. K. Eckert \& Company, Inc., Sarasota, Florida, 1995. 